\title{
Discrimination-shift behavior as a function of rule learning and the number of irrelevant categories
}

\author{
GEORGE W. WATSON \\ California State University, Fullerton, California 92634
}

\begin{abstract}
Reversal (R) and extradimensional (ED) choices in an optional discrimination shift study were investigated in college students $(N=150)$ as a function of varying amounts of previous mandatory $R$ or ED training and the number of irrelevant categories during preshift training. The findings were that as the number of training problems increased, $R$ or ED, the probability increased that the subject's optional choice was consistent with his previous shift training. Optional choice behavior was not influenced by the number of irrelevant categories.
\end{abstract}

The developmental mediational theory (Kendler \& Kendler, 1962, 1968, 1970a; Kendler, 1971) postulates that ontogenetic changes in discrimination shift behavior (Kendler \& Kendler, 1970b; 1972) reflect age-related capacities to represent symbolically the stimuli of each of the two sorting categories. The present experiment, conducted within that theoretical framework, investigates the influence of two procedural variables on the discrimination shift behavior of adults: (1) the amount of prior training to execute reversal (R) or extradimensional (ED) shifts and the number of irrelevant categories.

\section{METHOD}

Experimental Design and Subjects

A 2 by 3 by 3 factorial design was used with two types of training problems ( $R$ or $\mathrm{ED})$, three degrees of preliminary training (zero, one or two training problems) and three levels of irrelevant categories (one, two, or three). The three groups receiving zero training problems were combined into one group, thus reducing the number of groups from 18 to 15 . Ten subjects, from introductory psychology classes at UCSB, were randomly assigned to each of the 15 groups thus making a total of 150 subjects.

\section{Experimental Material}

Words from 24 natural language concepts were used as stimulus material (Cohen, Bousfield, \& Whitemarsh, 1957). The words were presented to the subjects on $3 \times 5$-in. index cards each containing a total of two, three, or four words. The number of words remained constant for each subject throughout the experimental session, but the position of each word varied randomly from card to card.

One of the words on each card represented a relevant category, while each of the remaining ones was an instance of an irrelevant category. Words of a relevant category were perfectly correlated with the correct choice response in a two-choice task while those of the irrelevant categories were uncorrelated with

This paper stems from the author's PhD thesis. For a more detailed report of the procedure and the results, the reader is referred to the original thesis (Watson, 1970).

Requests for reprints should be sent to George W: Watson, Department of Psychology, California State University, Fullerton, California 92634. the correct response. Each card contained only one instance from either one of the two relevant categories.

\section{Experimental Procedure}

The subject was given a practice problem immediately prior to the experimental task. Each stimulus card contained three words, one relevant, two irrelevant. The practice problem was completed when subject responded correctly without any assistance from the experimenter for eight successive cards. During the practice problem the experimenter emphasized two procedural principles: (1) the position of the word on a card played no role in determining what response was correct, and (2) only one word from a relevant category was on any particular card.

Following the practice problem, the subject was told that the experiment would begin and that he would be confronted with a similar task with different words. The training problems were mandatory shift problems in which the subject had to execute either a R- or ED-shift depending upon the group to which he was assigned. The criterion of preshift and postshift learning was correct responding for two consecutive blocks of trials in which all of the 12 stimulus cards for each problem appeared twice. The subject was given no clue of the impending shift at the end of the preshift training.

Following the completion of the last mandatory training problem the subjects were given an optional-shift discrimination problem (Kendler, Kendler, \& Learnard, 1962). Whereas in a mandatory shift problem the subject is required to execute a Ror ED-shift, an optional shift allows the subject to choose between responding in a $\mathrm{R}$ or ED manner. This is accomplished by an experimental procedure involving a sequence of three successive stages. The subject in the first stage learns to make the appropriate choice response to the instances of each relevant conceptual category. In the second stage, the optional phase, a switch occurs in the reinforcement contingencies with the correct responses being appropriate to both a R- and an ED-shift. To discover the basis on which he reached the criterion of learning in the optional stage, the subject is confronted with the third (test phase) stage in which the responses consistent with a R- or ED-shift are opposed to each other. Cards are used in which the pairing of the categories used in the optional stage are switched. This makes it possible to determine whether the subject was responding during the optional stage on the basis of a R- or ED-shift.

The criterion of learning in the preshift and optional-shift stage was two consecutive blocks of trials while during the test phase the subject was instructed to sort a block of 12 cards in the same manner as was done previously (i.e., during the optional-shift stage). During the test stage, the subject was told 
Table 1

Number of R-Responders in Test Stage of the Optional-Shift Problem as a Function of Prior R-Training and Number of Irrelevant Categories (Maximum $\mathbf{N}$ for Each Cell Equals 10)

\begin{tabular}{ccrrc}
\hline $\begin{array}{c}\text { N of } \\
\text { R-Training } \\
\text { Problems }\end{array}$ & \multicolumn{4}{c}{ Pairs of } \\
Irrelevant Categories & \\
1 & 1 & 2 & 3 & Mean \\
\hline 0 & 6.0 & 6.0 & 5.0 & 5.3 \\
1 & 8.0 & 8.0 & 8.0 & 8.0 \\
2 & 9.0 & 10.0 & 9.0 & 9.3 \\
Mean & 7.7 & 8.0 & 7.3 & 7.7 \\
\hline
\end{tabular}

that no feedback would be given as to the correctness of his choices.

\section{RESULTS AND DISCUSSION}

The prediction that a discrimination-shift problem-solving set, either $\mathrm{R}$ or $\mathrm{ED}$, would be established by prior training is confirmed by the data reported in Tables 1 and 2. These tables report the proportion of subjects who responded in an optional $\mathrm{R}$ (Table 1) or ED (Table 2) fashion after receiving a different number (zero, one, and two) of mandatory $\mathrm{R}$ or ED training problems.

All subjects responded either in a consistent $\mathrm{R}$ or ED fashion to all 12 cards of the test phase. As the number of mandatory training problems, either $\mathrm{R}$ or $\mathrm{ED}$, increased from zero to two, the proportion of optional-shift choices consistent with the training also increased. These data were analyzed with Page's L technique for ordered means (1963). The value of $L$ for the effects of mandatory $R$ and ED training are $L=42$, $\mathrm{p}<.01$ and $\mathrm{L}=41, \mathrm{p}<.01$, respectively. These findings support the notion that subjects can learn a discrimination-shift rule in the mandatory shift problems which transfer to the optional-shift task.

The results reported in Tables 1 and 2 indicate that the number of irrelevant categories does not influence choice response in discrimination shift problems, thus suggesting that postshift choices are guided solely by the preshift mediated representational response or some previously learned $\mathrm{R}$ or ED rule.

Although the present results indicated that the number of irrelevant categories do not effect $\mathrm{R}$ or ED choices, they do suggest that these choices can be influenced by the manner in which the irrelevant categories are displayed and/or by whether the subject is encouraged to attend to all of them. In an optional-shift task involving geometrical patterns containing binary values of two dimensions (e.g., color-form) $85 \%$ of college students chose an R-shift (Kendler \& Kendler, $1970 \mathrm{~b}$ ) whereas in the present experiment in which the
Table 2

Number of ED-Responders in Test Stage of Optional-Shift Problem as a Function of Prior ED-Training and Number of Irrelevant Categories (Maximum $\mathrm{N}$ for Each Cell Equals 10)

\begin{tabular}{crrrc}
\hline N of & \multicolumn{4}{c}{ Pairs of } \\
ED-Training & \multicolumn{1}{c}{ Irrelevant Categories } & \\
Problems & 1 & 2 & 3 & Mean \\
\hline 0 & 4.0 & 4.0 & 5.0 & 4.7 \\
1 & 7.0 & 8.0 & 8.0 & 7.7 \\
2 & 10.0 & 9.0 & 7.0 & 8.7 \\
Total & 7.0 & 7.0 & 6.7 & 6.9 \\
\hline
\end{tabular}

stimulus categories (dimensions) were physically separated and the experimenter directed the subject's attention to the various conceptual categories only 53\% behaved similarly. This minimizing of the relative difference between R- and ED-shifts is consistent with Johnson's (1966) failure to find any difference in the speed of R-and ED-shifts among college students when the various possible sorting categories were described to them prior to the experiment.

In sum, the present results demonstrate in adult human subjects (1) that discrimination shift rules, either $\mathrm{R}$ or ED, can be learned, (2) the number of irrelevant categories (dimensions) does not influence optional discrimination choices. In addition, the results suggest that the incidence of $\mathrm{R}$ choices in an optional-shift design can vary as a function of procedural variables such as the display of the stimulus material and the experimental instructions.

\section{REFERENCES}

Cohen, B. H., Bousfield, W. A., \& Whitmarsh, G. A. Cultural norms of verbal items in 43 categories. Technical Report No. 22, ONR Contract Nonr-631 (00), University of Connecticut, Storrs, Connecticut, 1957.

Johnson, P. Factors affecting transfer in concept ID problems. Journal of Experimental Psychology, 1966, 72, 655-660.

Kendler, H. H. Environmental and cognitive control of behavior. American Psychologist, 1971, 26, 962-973.

Kendler, H. H., \& Kendler, T. S. Vertical and horizontal processes in problem solving. Psychological Review, 1962, 69, 1-16.

Kendler, H. H., \& Kendler, T. S. Mediation and conceptual behavior. In K. W. Spence and J. T. Spence (Eds.), Psychology of learning and motivation. New York: Academic Press, Vol. 2 1968.

Kendler, H. H., \& Kendler, T. S. Developmental processes in discrimination learning. Human Development, 1970, 13, 65-89.

Kendler, T. S, \& Kendler, H. H. An ontogeny of optional-shift behavior. Child Development, 1970, 41, 1-27.

Kendler, T. S., Kendler, H. H., \& Learnard, B. Mediated responses to size and brightness as a function of age. American Journal of Psychology, 1962, 75, 571-586.

Page, E. B. Ordered hypotheses for multiple treatments: A significance test for linear ranks. Journal of American Statistical Association, 1963, 58, 216-230.

Watson, G. W., II. A theoretical and empirical analysis of discrimination-shift behavior. PhD thesis, University of California, Santa Barbara, 1970.

(Received for publication October $7,1974$. 